\title{
Efficacy of varicella vaccine in patients with solid tumours
}

\author{
R B HEATH, J S MALPAS, H O KANGRO, A WARD, J M MCENIERY, AND J E KINGSTON \\ Departments of Virology and Oncology, St Bartholomew's Hospital, London
}

SUMMARY Thirty nine children with malignant disease and without antibodies to varicella zoster virus were immunised with a live Oka strain varicella vaccine. Seroconversion was shown in 24 of those who were vaccinated but five of 18 who responded to the vaccine who were followed up for over six months subsequently lost their antibodies. Of 10 children who were revaccinated, nine responded to the second dose but three lost their antibodies within six months to two years. Four children developed reactions related to the vaccine, one local and three generalised, but all these were transitory and of no clinical concern. Nine of those who were vaccinated, including four who did not respond to the vaccine, have subsequently had close exposure to varicella but none has developed the disease.

Varicella is usually a mild disease that is rarely associated with serious complications. The important exception to this is when the disease occurs in those who are immunocompromised. In these circumstances the infection may disseminate to organs such as the lungs and brain and has been associated with mortalities ranging from $7 \%$ to $30 \% .{ }^{12}$ In 1974 Japanese workers described the development of a live varicella vaccine, ${ }^{3}$ and this was soon followed by a further report that the vaccine had been safely administered to children with leukaemia and other malignant diseases. ${ }^{2}$ Vaccines derived from the original Japanese Oka strain of varicella zoster virus have since been administered to various groups of children at risk from complications of varicella in trials carried out in both Japan and the United States. ${ }^{+6}$

We report our experience with one of these vaccines administered to children with malignant disease.

\section{Patients and methods}

Patients. Children seronegative for antibodies to varicella zoster virus were selected for vaccination from patients who were attending the paediatric oncology clinic at our hospital. The underlying malignancies in these children are shown in the Table.

The vaccine was not given to children with disseminated disease or to those whose lymphocyte count was less than $1.2 \times 10^{\%} / 1$. Informed consent was obtained from the parents of all the children before vaccination was undertaken and the study was approved by the ethical committee of our hospital.

Vaccination. The Smith Kline-RIT live Oka strain of varicella vaccine was kindly provided by $\mathrm{Dr} F$ Andre (RIT, Rixensart, Belgium). A single $1 \mathrm{ml}$ dose of the vaccine, containing not less than 1000 plaque forming units, was administered subcutaneously. Some children were vaccinated on completion of treatment for their disease, whereas others were given vaccine between courses of chemotherapy. In either instance vaccine was not given until 10 days had elapsed since the last treatment and treatment was not started until at least 10 days had elapsed after vaccination.

Table Malignancies in 39 patients given live varicella vaccine

\begin{tabular}{ll}
\hline Rhabdomyosarcoma & 9 \\
Wilms' tumour & 9 \\
Non-Hodgkin's lymphoma & 4 \\
Ewing's tumour & 3 \\
Acute lymphocytic leukaemia & 3 \\
Central nervous system tumour & 2 \\
Nasopharyngeal carcinoma & 2 \\
Yolk sac tumour & 2 \\
Osteosarcoma & 2 \\
Histiocytosis & 1 \\
Renal carcinoma & 1 \\
Acute myeloid leukacmia & 39 \\
\hline Total & \\
\hline
\end{tabular}


Follow up. Blood samples were taken routinely, from each patient before vaccination and five weeks after vaccination for evidence of seroconversion. Thereafter, blood samples were taken at each attendance at the clinic to determine the longevity of any induced humoral response. Parents were asked to report immediately any reaction to the vaccine and at each attendance at the clinic they were questioned carefully about any exposure to varicella.

Serology. Serum samples before and after vaccination were tested for antibodies to varicella zoster virus by complement fixation and radioimmunoassay procedures that have been described previously. ${ }^{78}$

\section{Results}

Serological response to the vaccine. To date 39 seronegative children have been vaccinated, and $24(62 \%)$ seroconverted within five weeks of vaccination. The antibody titres after vaccination have shown considerable individual variation, ranging from $<4$ to 512 (median:8) by complement fixation and 100 to $>25600$ (median:3200) by radioimmunoassay. Antibodies in four patients were detectable only by radioimmunoassay and not by the less sensitive complement fixation test. One of these patients had received zoster immune globulin because of exposure to varicella three days after vaccination, so the antibodies detected may not have been induced by the vaccine.

Interestingly, three of those who were vaccinated who had not initially seroconverted were later found to have developed antibodies to varicella zoster virus. Two of these were found to have good antibody titres six and 11 months, respectively, after vaccination and both had been exposed to varicella in the interval between the tests. Neither had developed any symptoms suggestive of varicella. The third patient was negative two years after vaccination but when retested at 3 years had antibody titres of 128 by complement fixation and $>25600$ by radioimmunoassay. There was no known exposure to varicella nor any symptoms suggestive of varicella during this period.

Reactions to the vaccine. Only four $(10 \%)$ of the children showed any reaction to the vaccine. Three patients developed very mild varicella about three weeks after vaccination, from which they rapidly recovered. The fourth child developed a small crop of vesicles at the inoculation site, which during the next few days became surrounded by a reddened indurated area of skin, $5 \mathrm{~cm}$ in diameter, with a necrotic centre (Fig. 1). This local lesion developed three weeks after vaccination and healed within seven days. These children with clinical reactions interestingly developed the highest antibody responses, comparable with those after wild virus infection.

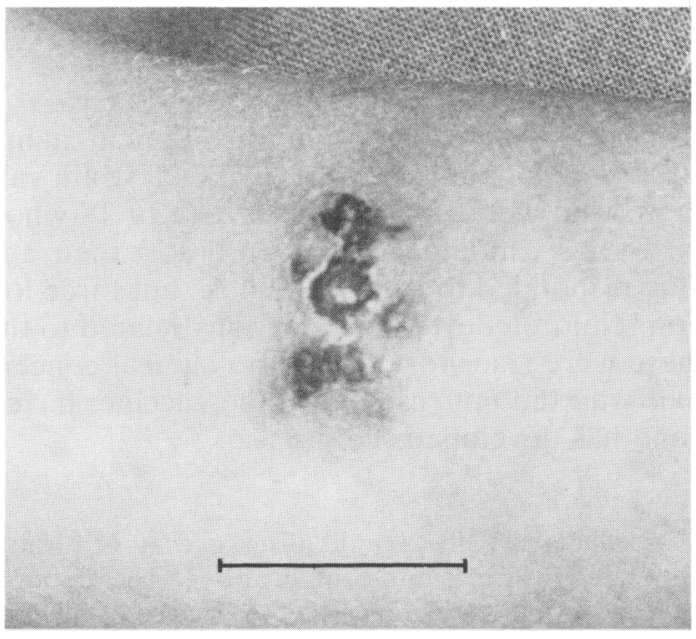

Fig. 1 Local reaction at the inoculation site on the right upper arm in a recipient of the live varicella vaccine. Bar is 5 $\mathrm{cm}$ in length.

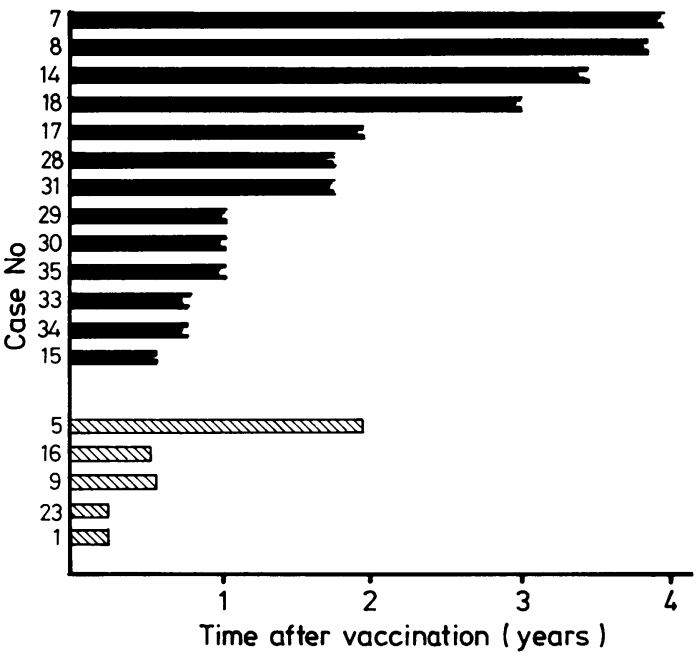

Fig. 2 Duration of immune response in patients who initially responded to vaccination. Solid bars represent patients with persisting antibody; hatched bars represent those who have lost their antibodies. 
Longevity of the humoral immune response. So far 18 of the 24 patients who developed antibodies after vaccination have been followed up for more than six months. It was not possible to obtain comparable data on the other patients because of death due to malignancy, recent vaccination, or loss to follow up. Figure 2 shows that $13(72 \%)$ of the 18 patients still had detectable antibody titres in all serum samples that were tested during intervals that ranged from six months to four years. The remaining five patients lost their antibodies after periods ranging from six months to two years.

There was no definite correlation between duration of antibody response and initial antibody titre after vaccination. One of the patients who had a vaccine reaction (case 5) developed one of the highest antibody titres initially but lost the antibodies by two years.

Protection. Altogether, nine of those who were vaccinated experienced close (home or playmate) contact with varicella. Five of these were patients who did respond to the vaccine and four who did not. None developed clinical varicella, although one of the responders showed a considerable rise in antibody titre after contact and two nonresponders seroconverted. It was of interest that two siblings of one of those who was vaccinated had had contact with the same index case and both developed varicella.

Revaccination. So far 10 children have been revaccinated six to 12 months after their first doses, five because of poor and waning initial responses and five who did not respond. Nine of these children responded to the second dose, but on follow up three $(33 \%)$ had lost their antibodies six months to two years after revaccination. Four children remained positive for at least one to three years.

There was no correlation between the responses after the first and second doses. None of those who were revaccinated has shown clinical responses.

\section{Discussion}

This investigation with the live Oka strain varicella vaccine encourages us to believe that it is of prophylactic value for children who are being treated for malignant disease. The few reactions observed in children with solid tumours were mild and of little concern to attending physicians. This is consistent with the observations made in trials of this vaccine in children with leukaemia. ${ }^{9}$ Immunocompromised children who react to this attenuated vaccine may be those that would have experienced the severe, perhaps fatal, forms of varicella had they been infected with wild strains.
The antibody responses to the vaccine were generally inferior to that observed after wild virus infection in normal children. This is, of course, a general feature of attenuated vaccines and could be expected particularly when administered to immunocompromised patients whose ability to produce antibody may be impaired. Indeed, one of our seronegative patients developed typical varicella, fortunately without complications, before she had been vaccinated. Three weeks after onset her antibody titres by complement fixation and radioimmunoassay were only 4 and 600 , respectively, and no antibody could be detected by either method in a serum sample taken a year later. Furthermore, one of the children who did not initially respond to the vaccine but seroconverted after contact with varicella lost the antibodies within nine months. The level of immune responsiveness in the recipient affects the seroconversion rate after vaccination. ${ }^{9}$ Thus the immunosuppressed state of our vaccines with solid tumours probably accounts for the considerably lower seroconversion rate $(61 \%)$ observed, compared with the rate in excess of $90 \%$ reported in healthy recipients. ${ }^{9}$

Despite the poor humoral response, the vaccine seems to confer good protection. Nine of our patients who were vaccinated were subsequently exposed to varicella and none $(<11 \%)$ developed the disease, although some may have had subclinical infection, which may explain the delayed but high antibody responses occasionally observed. In a large study, however, involving 192 children with leukaemia, $4 \%$ of those who were vaccinated who received one dose of vaccine subsequently developed varicella. ${ }^{6}$ These findings should be compared with the expected infection rate in susceptible children of at least $80 \%$ after close contact. The apparent protective effect even in patients with poor antibody responses shows the difficulty in determining immune state in immunocompromised patients. Even a sensitive antibody test such as radioimmunoassay may still be inadequate for showing immunity in these patients, and a test for cellular immunity -for example, lymphocyte transformation ${ }^{10}$ - may be more suitable.

As a result of the unsatisfactory antibody response in some of our children we have begun revaccinating those who either failed to seroconvert or who no longer have detectable antibody. We are encouraged by our early results with this new regimen in that nine of 10 patients who have been revaccinated have developed good antibody responses, although three have subsequently lost their antibodies again. Clearly, further studies are required to determine the optimum immunisation schedule in these patients. 


\section{Heath, Malpas, Kangro, Ward, McEniery, and Kingston}

We believe that sufficient evidence about varicella vaccine has now been accumulated over the past 10 years, ${ }^{9}$ regarding its safety and efficacy, to recommend its wider use in patients at risk of developing the severe complications of varicella.

We acknowledge the help of Dr A Smith and Miss V Potter with this study.

\section{References}

' Feldman S, Hughes WT, Daniel CB. Varicella in children with cancer: 77 cases. Pediatrics 1975;56:388-97.

2 Izawa T, Ihara T, Hattori A, Iwaza T. Application of a live varicella vaccine in children with acute leukaemia or other malignant diseases. Pediatrics 1977;60:805-9.

3 Takahashi M, Otsuka T, Okuno Y, et al. Live vaccine used to prevent the spread of varicella in children in hospitals. Lancet $1974 ;$ ii: $1288-90$.

4 Ha K. Baba K. Ikeda T, et al. Application of a live varicella vaccine to children with acute leukaemia or other malignancies without suspension of anticancer therapy. Pediatrics 1980;65:346-50.

s Brunnell PA, Shehab Z, Geiser C, Waugh JE. Administration of live varicella vaccine to children with leukaemia. Lancet 1982;ii:1069-73.

${ }^{6}$ Gershon AA, Steinberg SP, Gelb L, et al. Live attenuated varicella vaccine. Efficacy for children with leukaemia in remission. JAMA 1984;252:355-62.

7 Ronalds CJ, Hardiman AE, Griffiths PD. Hotting up the complement fixation test. J Hyg (Camb) 1983;90:127-34.

* Campbell-Benzie A, Kangro HO, Heath RB. The development and evaluation of a solid phase radioimmunoassay (RIA) procedure for the determination of susceptibility to varicella. J Virol Methods 1981;2:149-58.

9 Andre FE. Worldwide experience with the Oka-strain live varicella vaccine. Postgrad Med J 1985;61:(Suppl 4):113-20.

10 Ndumbe PM, Cradock-Watson JE, MacQueen S, et al. Immunisation of nurses with a live varicella vaccine. Lancet 1985:i:1144-7.

Correspondence to Professor R B Heath, Department of Virology, St Bartholomew's Hospital, West Smithfield, London EC1A 7BE.

Received 9 January 1986 